\title{
AVALIAÇÃO DA CONFIABILIDADE EM SISTEMAS DE DISTRIBUIÇÃO CONSIDERANDO FALHAS DE GERAÇÃO E TRANSMISSÃO
}

\author{
A.M. Cassula* \\ romeu@eletro.ufrgs.br \\ L.A.F. Manso ${ }^{\dagger}$ \\ edson@lcmi.ufsc.br \\ A.M. Leite da Silva* \\ teel@ece.ucsb.edu \\ R. Billinton ${ }^{\ddagger}$ \\ edson@lcmi.ufsc.br \\ ${ }^{*}$ Grupo de Engenharia de Sistemas - GESis, Universidade Federal de Itajubá - UNIFEI, MG, Brasil \\ ${ }^{\dagger}$ Departamento de Eletricidade - DEPEL, Universidade Federal de São João del Rei - FUNREI, MG, Brasil \\ $\ddagger$ Power System Research Group - University of Saskatchewan - Saskatoon, Canada
}

\section{ABSTRACT}

This paper presents a new methodology to evaluate the reliability of distribution systems considering the impact of failures from the generation and transmission (G\&T) systems. Therefore, an integrated adequacy evaluation, including generation, transmission and distribution, is performed in order to provide a more detailed information about interruptions experienced by consumers. The G\&T systems are represented by a fictitious equivalent network, whose parameters are obtained by Monte Carlo non-sequential simulation. The equivalent G\&T network is then connected with the distribution network and analyzed by the minimal cut-set theory. Traditional distribution indices (e.g. SAIFI, SAIDI, etc.) as well as the LOLC - Loss of Load Cost - indices are disaggregated to measure the contribution of G\&T and distribution systems on the overall system indices. The proposed method is applied to the IEEE-RTS, which represents the G\&T system, connected with the RBTS, which represents the distribution system. The results and their potential applications to the new power system competitive environment are discussed.

Artigo submetido em 13/12/2000

1a. Revisão em 19/6/2002; 2a. Revisão 11/10/2002

Aceito sob recomendação do Ed. Assoc. Prof. José L. R. Pereira
KEYWORDS: Distribution reliability, reliability worth, generation transmission and distribution reliability, hierarchical level 3.

\section{RESUMO}

Este artigo apresenta uma nova metodologia para a análise da confiabilidade de sistemas de distribuição, onde se considera os impactos das falhas provenientes dos sistemas de geração e transmissão. Portanto, é viabilizada uma avaliação integrada, incluindo geração, transmissão (G\&T) e distribui-ção, de modo a produzir uma informação mais detalhada sobre a causa das interrupções experimentadas pelos consumidores. O sistema G\&T é representado por uma rede equivalente fictícia, cujos parâmetros são obtidos através de simulação Monte Carlo não-seqüencial. Esta rede equivalente G\&T é conectada ao sistema de distribuição, que é então analisado utilizando a teoria dos conjuntos mínimos de corte. Índices de distribuição tradicionais (e.g. FEC, DEC, etc.) bem como a LOLC (Loss of Load Cost - Custo Esperado da Perda de Carga), são desagregados com o intuito de quantificar a contribuição dos sistemas G\&T e distribuição em relação ao sistema total. O método proposto é testado em um sistema constituído pelo sistema de geração e transmissão IEEE-RTS, conectado ao sistema de distribuição IEEE-RBTS. Os resultados e suas po- 
tenciais aplicações para o novo cenário competitivo dos sistemas elétricos são discutidos.

PALAVRAS-CHAVE: Confiabilidade em sistemas de distribuição, valor da confiabilidade, confiabilidade na geração transmissão e distribuição, nível hierárquico 3 .

\section{INTRODUÇÃO}

Os sistemas de distribuição (Billinton e Allan, 1994; Billinton, 1988; Allan e da Silva, 1995; Billinton e Satish, 1996; Chowdhury e Koval, 1998; Allan et alii, 1991; Billinton e Jonnavithula, 1996) sempre receberam pouca atenção em relação às técnicas de avaliação de confiabilidade quando comparados aos sistemas de geração e transmissão (Leite da Silva et alii, 1991; Melo et alii, 1993; Goel e Billinton, 1993; Wenyuan e Billinton, 1993; Mello et alii, 1994; Mello et alii, 1997; Leite da Silva et alii, 2000; Manso et alii, 1999). Dois argumentos têm sido utilizados: sistemas G\&T necessitam de gastos vultuosos e podem causar conseqüências catastróficas tanto para a sociedade quanto para seu próprio ambiente. Análises estatísticas realizadas pelas concessionárias de energia sobre falhas nos consumidores, demonstram que os sistemas de distribuição são responsáveis pela maior parte das contribuições individuais que acarretam indisponibilidade de fornecimento para os consumidores (Billinton e Allan, 1994). Além disso, embora um determinado reforço ou um novo esquema de proteção em sistemas de distribuição tenha um custo relativamente barato, coletivamente os investimentos podem atingir níveis significativos.

Nos últimos anos, os sistemas de distribuição têm recebido uma atenção especial, principalmente devido ao processo de restruturação e privatização do setor elétrico. Neste novo cenário, as companhias de distribuição serão responsáveis pela venda de todo tipo de serviço associado com os sistemas de distribuição e, portanto, estarão em busca por maior eficiência, no sentido de maximizar seus lucros, mantendo a qualidade de serviço em conformidade com as normas impostas pelas agências reguladoras. Com isso, o valor da confiabilidade deverá ser corretamente avaliado e inserido nas tarifas para possíveis indenizações aos consumidores, no caso de haver interrupções.

A avaliação integrada da confiabilidade de um sistema elétrico de potência incluindo geração, transmissão e distribuição (conhecido como Nível Hierárquico 3, ou simplesmente NH3) é uma importante meta para o planejamento e operação de sistemas de potência (Leite da Silva et alii, 1991; Melo et alii, 1993). Os níveis hierárquicos usuais (Billinton e Allan, 1988) estão repre-

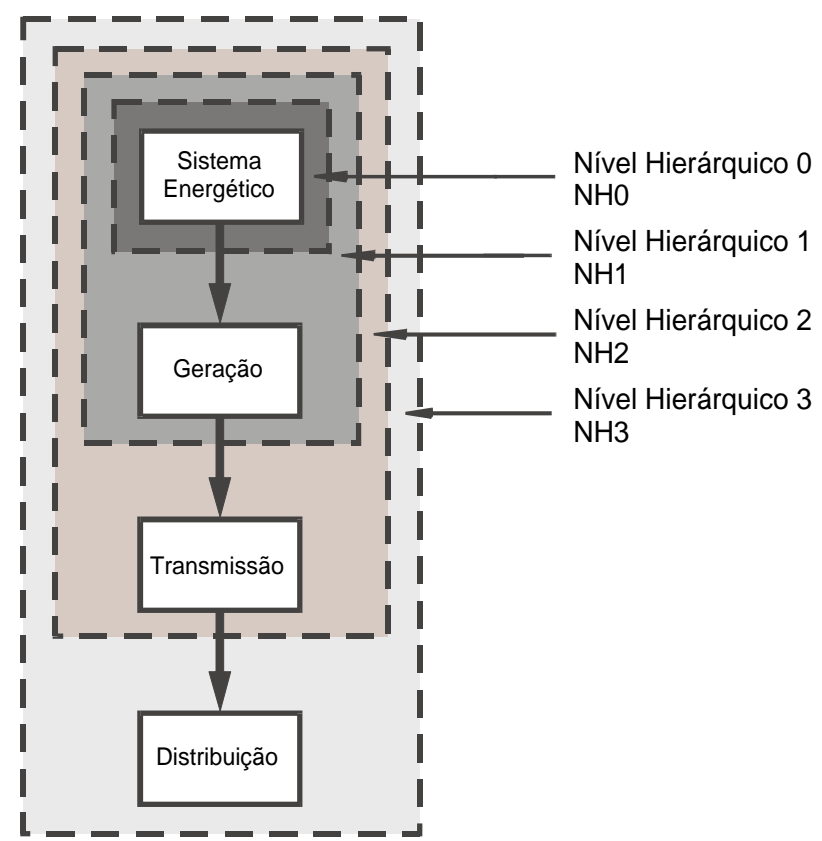

Figura 1: Níveis Hierárquicos de um Sistema de Potência

sentados na Figura 1. Entretanto, devido à dimensão do problema, estudos de confiabilidade em sistemas de distribuição têm sido realizados considerando o sistema G\&T representado por pontos de fornecimento com capacidade ilimitada e 100\% confiáveis.

Neste trabalho, uma nova metodologia é proposta para avaliar o impacto das falhas do NH2 nos sistemas de distribuição. Isto será obtido por uma simulação Monte Carlo não-seqüencial e pode envolver diferentes barras do NH2. Em função da política de corte de carga no nível de distribuição, uma rede fictícia é construída de modo que a disposição dos componentes simulem as interrupções oriundas do NH2. Este procedimento generaliza os conceitos propostos em (Billinton e Satish, 1996). O sistema de distribuição juntamente a rede fictícia são analisados utilizando os conceitos de minimal cut-set (Billinton e Allan, 1992). Além dos índices usuais de confiabilidade para o sistema e pontos de carga, outro índice, denominado LOLC - Loss of Load Cost (Allan e da Silva, 1995; Chowdhury e Koval, 1998; Goel e Billinton, 1993; Wenyuan e Billinton, 1993; Mello et alii, 1994; Mello et alii, 1997; Leite da Silva et alii, 2000; Manso et alii, 1999; Billinton e Allan, 1988), será avaliado e desagregado considerando os níveis hierárquicos. Portanto, a metodologia proposta define, do ponto de vista econômico, as responsabilidades sobre possíveis prejuízos causados devido às interrupções. 


\section{CONFIABILIDADE DE SISTEMAS DE DISTRIBUIÇÃO}

Um sistema de distribuição pode ser representado por uma rede cujos componentes podem estar conectados em série, em paralelo, ou ainda por uma combinação qualquer dos componentes. Existem vários métodos disponíveis para a solução e avaliação destas redes (Billinton e Allan, 1992). Porém, quando se está analisando continuidade de fornecimento, o método dos conjuntos mínimos de corte é o que melhor se aplica, pois indica diretamente as falhas predominantes e, portanto, reflete o comportamento distinto dos modos de falha do sistema.

\subsection{Técnicas de Avaliação}

O processo de Markov e a abordagem de freqüência e duração formam um excelente método de modelagem e análise para aplicações da confiabilidade (Billinton e Allan, 1992). Para sistemas maiores e mais complexos, como redes de distribuição, foram desenvolvidas aproximações baseadas nas equações que derivam deste método. Estas equações podem ser empregadas conjuntamente com a teoria dos conjuntos mínimos de corte, pois fornecem resultados precisos com uma maior rapidez, para a maioria dos sistemas de distribuição que se encontram na prática. Neste caso, a rede de confiabilidade consiste em um número de minimal cut-sets conectados em série e cada cut-set é constituído por componentes conectados em paralelo, como mostra a Figura 2. Para o cálculo, inicialmente utilizam-se as equações do sistema paralelo para cada cut-set, então combinam-se estes índices através das equações do sistema série, para enfim determinar os índices equivalentes.

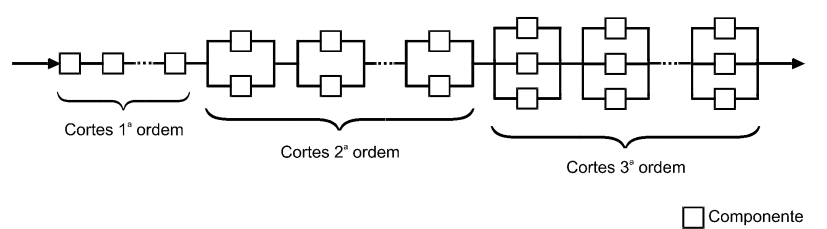

Figura 2: Rede Equivalente de Confiabilidade

\section{2 Índices de Desempenho}

Para as companhias de eletricidade é essencial dividir o sistema de distribuição em níveis de confiabilidade, e definir índices para atender sua função básica de fornecer energia confiável ao menor custo, para todos os setores da sociedade. Este procedi-mento é conhecido como avaliação do desempenho passado, ou histórico, da confiabi- lidade, o qual é utilizado pela maioria das empresas. A análise de desempenho futuro, ou a avaliação preventiva da confiabilidade, é uma outra filosofia que possibi-lita determinar reforços os necessários ao sistema e comparar alternativas de expansão. Contudo, para quantificar o desempe-nho passado ou futuro do fornecimento de energia nos pontos de carga dos consumidores e para o sistema total, os índices a seguir são os mais empregados (Billinton e Allan, 1994):

\section{Índices para Pontos de Carga: \\ FIC Freqüência de Interrupção [falhas/ano] Indivi-dual por Unidade Consumidora \\ DIC Duração de Interrupção Indi- [horas/ano] vidual por Unidade Consumi- dora \\ r Duração da Falha [horas] \\ EENS Energia Esperada Não Su- [kWh/ano] prida \\ Índices de Sistema: \\ FEC Freqüência Equivalente de Interrupção por Unidade Consumidora \\ [Interrupções/consumidor ano] \\ DEC Duração Equivalente de Interrupção por Unidade Consumidora \\ [horas/consumidor ano]}

No Nível Hierárquico 2, o emprego da avaliação preventiva é bastante difundida para planejar a confiabilidade do sistema. Entretanto, no NH3 esta técnica não é tão popular quanto ao desempenho passado da confiabilidade. Com a abertura da competitividade no setor, está aumentando o interesse por metodologias de otimização econômica de planejamento e expansão na distribuição. Em um futuro próximo, será necessário que todas companhias de distribuição identifiquem quais os pontos no sistema que devem receber a prioridade nos investimentos. Portanto, o desempenho futuro será uma informação valiosa no processo de tomada de decisões para sistemas de distribuição.

A confiabilidade de qualquer serviço elétrico, incluindo a atividade de distribuição, deve ser baseada no balanço dos custos para a concessionária e o valor dos benefícios oferecidos aos consumidores. Um valor de referência da confiabilidade para ser utilizado no planejamento (Chowdhury e Koval, 1998; Burns e Gross, 1990) deve considerar uma solução de mínimo custo, onde o custo total inclui custos de investimento, custos operacionais e custos de interrupção. Portanto a LOLC, que representa o custo de interrupção, se tornará o mais importante índice que representa a confiabilidade no planejamento de 
sistemas elétricos.

Uma comparação de métodos alternativos para a avaliação do índice LOLC em sistemas de geração e transmissão está descrito em (Billinton e Allan, 1988). Este índice depende basicamente dos custos unitários (UC Unit Interruption Cost) de interrupção de cada classe de consumidores, usualmente fornecido em US\$/kWh. Tais custos são obtidos através de estudos econômicos específicos (levantados junto aos consumidores). Estes estudos apresentam diferentes fatores que influenciam na formação dos UCs, sendo a duração da interrupção considerado o fator mais importante (EPRI, 1989). Portanto, o nível de exatidão estabelecido para determinar a duração da interrupção interfere diretamente na qualidade da estimativa do índice LOLC.

\section{IMPACTOS DAS FALHAS DE G\&T NO SISTEMA DE DISTRIBUIÇÃO}

Existem vários benefícios associados com uma avaliação completa da confiabilidade. Os índices globais fornecem uma estimativa da confiabilidade até o nível dos consumidores, e podem ser usados para estimar a contribuição que cada zona funcional exerce em um determinado ponto de carga e, portanto, otimizar a alocação de recursos (Billinton e Jonnavithula, 1996).

O cálculo dos índices globais pode ser dividido em três etapas principais. Primeiramente, é usado um algoritmo capaz de avaliar o sistema G\&T, com o intuito de gerar um número suficiente de amostras que produzem interrupções na barra (ou barras) de alta tensão do sistema de distribuição. Neste trabalho, a avaliação do NH2 foi realizada por uma simulação Monte Carlo nãoseqüencial. Na segunda etapa, serão extraídos parâmetros do processo de simulação anterior para auxiliar na construção de uma rede equivalente, que também depende da política de corte de carga utilizada no sistema de distribuição. Uma vez definida a rede G\&T fictícia, esta será conectada à rede de distribuição, constituindo assim a terceira e última etapa da análise NH3.

\subsection{Parâmetros que Caracterizam as Falhas de G\&T}

Para se definir, do ponto de vista da confiabilidade, um componente fictício que pertença a rede equivalente G\&T, é necessário apenas conhecer sua taxa de falha $(\lambda)$ e sua indisponibilidade $(U)$. Uma metodologia para determinar tais componentes fictícios será descrita a seguir, considerando um sistema de distribuição com $N$ alimentadores (ramais).
Durante a convergência da simulação Monte Carlo, uma série de eventos que representam os estados de falha associados com a barra da alta tensão conectada à rede de distribuição são armazenados. Cada estado de falha é caracterizado pelos seguintes parâmetros: frequiência incremental $\left(f_{\text {inc }}\right)$ e quantidade de carga a ser cortada devido a falhas no G\&T $\left(C C_{G T}\right)$. Os cortes de carga são agrupados em intervalos de potências que correspondem ao total de cargas conectadas aos ramais, como ilustra a Tabela 1 .

Nesta Tabela, $N I$ é o Número do Intervalo e $P_{R 1}, P_{R 2}$, $P_{R 3} \ldots P_{R k} \ldots P_{R N}$ são as potências totais referentes aos ramais $1,2,3 \ldots k \ldots \quad N$. O número de intervalos é também o número de ramais conectados na barra de alta tensão. Este procedimento pode ser facilmente estendido para sistemas de distribuição que possuem mais de uma barra como entrada.

A probabilidade ou indisponibilidade $(U)$ e a freqüência associada a cada intervalo de potência (que corresponde ao corte de carga ou evento de falha), podem ser obtidas pelas Eqs. (1), abaixo. Observa-se que a freqüência de falha é aproximadamente a pseudo taxa de falha associada com os componentes fictícios. Portanto, os componentes G\&T fictícios são totalmente caracterizados por $\lambda$ e $U$.

$$
U_{k}=P_{k}=\frac{N_{k}}{N T} ; \quad \lambda_{k} \cong f_{k}=\frac{\sum_{j=1}^{N_{k}} f_{i n c_{j}}}{N T} ; \quad r_{k}=\frac{U_{k}}{\lambda_{k}}
$$

onde,

$P_{k}=$ Probabilidade do intervalo de potência $k$.

$U_{k}=$ Indisponibilidade do intervalo de potência $k$.

$N_{k}=$ Número total de estados (de falha)pertencentes ao intervalo de potência $k$.

$N T=$ Número total de simulações realizadas.

$\lambda_{k}=$ Taxa de falha do intervalo de potência $k$.

$f_{k}=$ Freqüência de ocorrência do intervalo de potência $k$.

$r_{k}=$ Duração média das falhas relativas ao intervalo de potência $k$.

$\sum_{j=1}^{N_{k}} f_{i n c_{j}}=$ Somatório da freqüência incremental dos estados $\mathrm{j}$ pertencentes ao intervalo de potência $k$.

Normalmente, o parâmetro $U$ é fornecido em [horas/ano], i.e.:

$$
U_{k}=P_{k} \times 8760 \quad[\text { horas } / \text { ano }]
$$


Tabela 1: Intervalo de Potência dos Componentes G\&T

\begin{tabular}{|c|c|c|c|}
\hline \multicolumn{2}{|c|}{ Ramal } & $N I$ & Intervalo de Potência \\
\hline R1 & $P_{R 1}$ & 1 & $0<C C_{G T} \leq P_{R 1}$ \\
\hline R2 & $P_{R 2}$ & 2 & $P_{R 1}<C C_{G T} \leq P_{R 1}+P_{R 2}$ \\
\hline$\vdots$ & $\vdots$ & $\vdots$ & $\vdots$ \\
\hline Rk & $P_{R k}$ & $\mathrm{k}$ & $P_{R 1}+P_{R 2}+\ldots+P_{(R k-1)}<C C_{G T} \leq P_{R 1}+P_{R 2}+\ldots+P_{R k}$ \\
\hline$\vdots$ & $\vdots$ & $\vdots$ & $\vdots$ \\
\hline $\mathrm{RN}$ & $P_{R N}$ & $N$ & $P_{R 1}+P_{R 2}+\ldots+P_{(R N-1)}<C C_{G T} \leq P_{R 1}+P_{R 2}+\ldots+P_{R N}$ \\
\hline
\end{tabular}

\subsection{Representação das Políticas de Corte de Carga}

Cada concessionária emprega uma política de corte de carga para seus sistemas de potência. A política adotada obedece a critérios que procuram reduzir os efeitos provocados por falhas no sistema e minimizar os custos de interrupção de energia. As políticas de corte de carga podem ser representadas ou modeladas através da disposição dos componentes G\&T fictícios dentro do sistema de distribuição. Para melhor ilustrar, considera-se um sistema de distribuição com 4 ramais: R1, R2, R3 e R4. A potência de cada ramal e a política de corte de carga adotada estão expressas na Tabela 2 .

Tabela 2: Política de Corte de Carga

\begin{tabular}{|c|c|c|c|}
\hline \multicolumn{2}{|c|}{$\begin{array}{c}\text { Potência do } \\
\text { Ramal [MW] }\end{array}$} & $\begin{array}{c}\text { Intervalo de } \\
\text { Potência }\end{array}$ & $\begin{array}{c}\text { Desconecta o } \\
\text { Ramal }\end{array}$ \\
\hline R1 & 20 & $0<C C_{G T} \leq 20$ & $\mathrm{R} 1$ \\
\hline R2 & 15 & $20<C C_{G T} \leq 35$ & $\mathrm{R} 1+\mathrm{R} 2$ \\
\hline R3 & 40 & $35<C C_{G T} \leq 75$ & $\mathrm{R} 1+\mathrm{R} 2+\mathrm{R} 3$ \\
\hline R4 & 25 & $75<C C_{G T} \leq 100$ & $\mathrm{R} 1+\mathrm{R} 2+\mathrm{R} 3+\mathrm{R} 4$ \\
\hline
\end{tabular}

A política de corte de carga adotada na Tab. 2, pode ser representada conectando os componentes G\&T fictícios, conforme a rede equivalente demonstrada na Figura 3. Neste exemplo, o Ramal 1 será desconectado se o corte de carga, devido a G\&T, estiver entre 0 e $20 \mathrm{MW}$. Porém, se a quantidade de carga a ser cortada estiver entre 20 e $35 \mathrm{MW}$, ambos os ramais R1 e R2 deverão ser desconectados, e assim por diante. Observe que os parâmetros $\lambda$ e $U$ relativos a estes componentes fictícios já foram obtidos nas considerações anteriores.

\section{AVALIAÇÃO INTEGRADA DA CONFI- ABILIDADE}

\subsection{Sistema Básico de Teste}

O sistema analisado é composto pelo sistema de distribuição RBTS-Barra2 (Allan et alii, 1991), conectado à Barra 6 do RTS (Task Force of the Application of

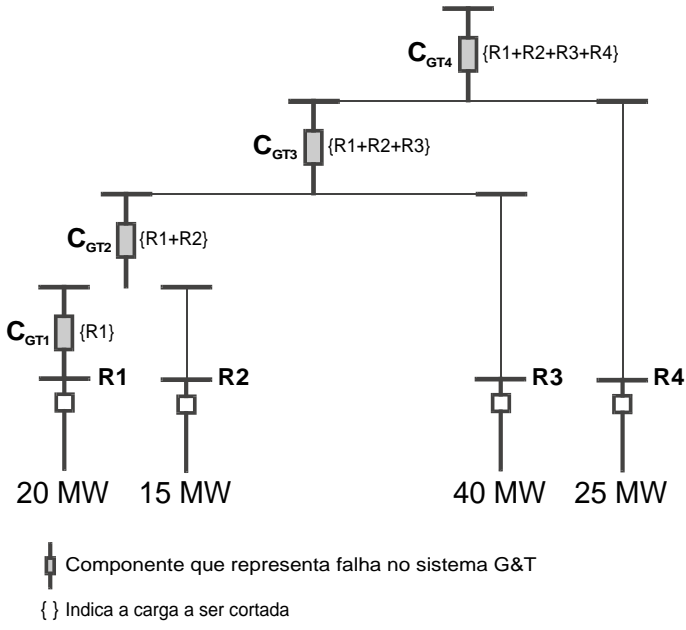

Figura 3: Rede que Representa a Política de C.C. da Tabela 2

Probability Methods Subcommittee, 1979). Linhas de transmissão aérea foram utilizadas ao invés de cabos. A Barra 2 do RBTS foi aqui renomeada para Barra 25. A carga média e de pico desta barra são respectivamente, 12,29 MW e 20 MW. Entretanto, existe a necessidade de adequar o nível de tensão, o que é feito através da inclusão de um transformador de $138 / 11 \mathrm{kV}$. A reatância deste transformador é igual a $0,12585 \mathrm{pu}(50 \%$ maior que os do RTS). A sua taxa de falha foi adotada como sendo igual a 0,02 [falhas/ano] e seu tempo médio de reparo (MTTR, Mean Time to Repair ou "r") de 768 horas. A carga média da Barra 6 é igual a $71,8 \mathrm{MW}$ e a carga de pico de 116,8 MW.

Para o cálculo do índice LOLC, tanto por barra quanto para o sistema, é necessário possuir os custos unitário de interrupção. Neste trabalho, foram utilizados os dados da Ontario Hydro para os setores industrial, comercial e residencial (Mello et alii, 1994; Mello et alii, 1997; Leite da Silva et alii, 2000; Manso et alii, 1999). Para o sistema de distribuição RBTS-Barra2, os tipos de con- 


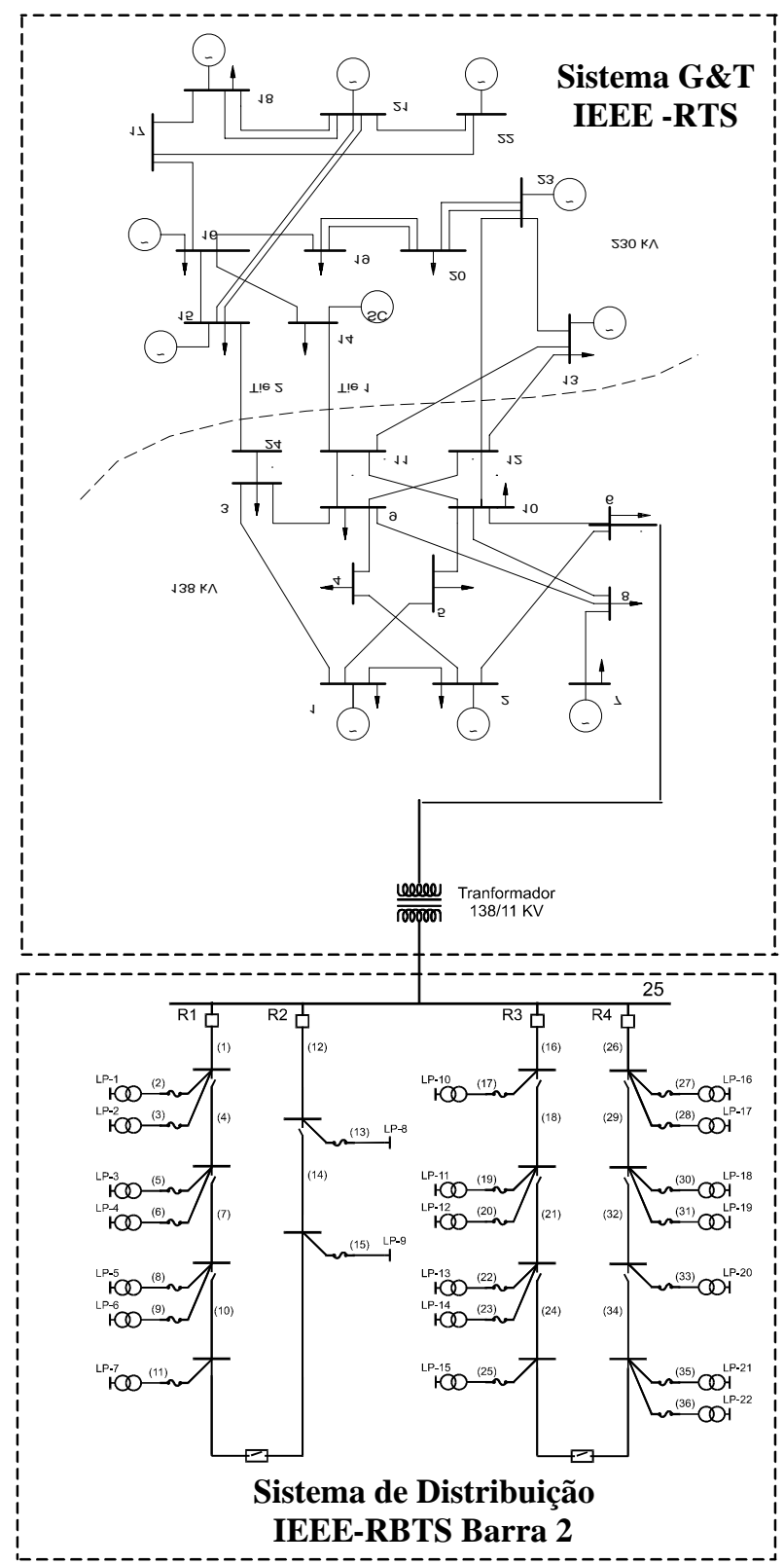

Figura 4: Sistema Elétrico de Potência Total - NH3

sumidores small user e government/institutions foram interpretados como industrial e comercial.

A política de corte de carga para a Barra 6 do sistema G\&T considera que a prioridade de corte será sobre o sistema de distribuição da Barra 25. Para o sistema de distribuição, primeiramente a análise será efetuada considerando a política de corte de carga apresentada na Tabela 2 e mostrada na Figura 3. Porém, a potência dos ramais são aquelas apresentadas para o sistema IEEE-

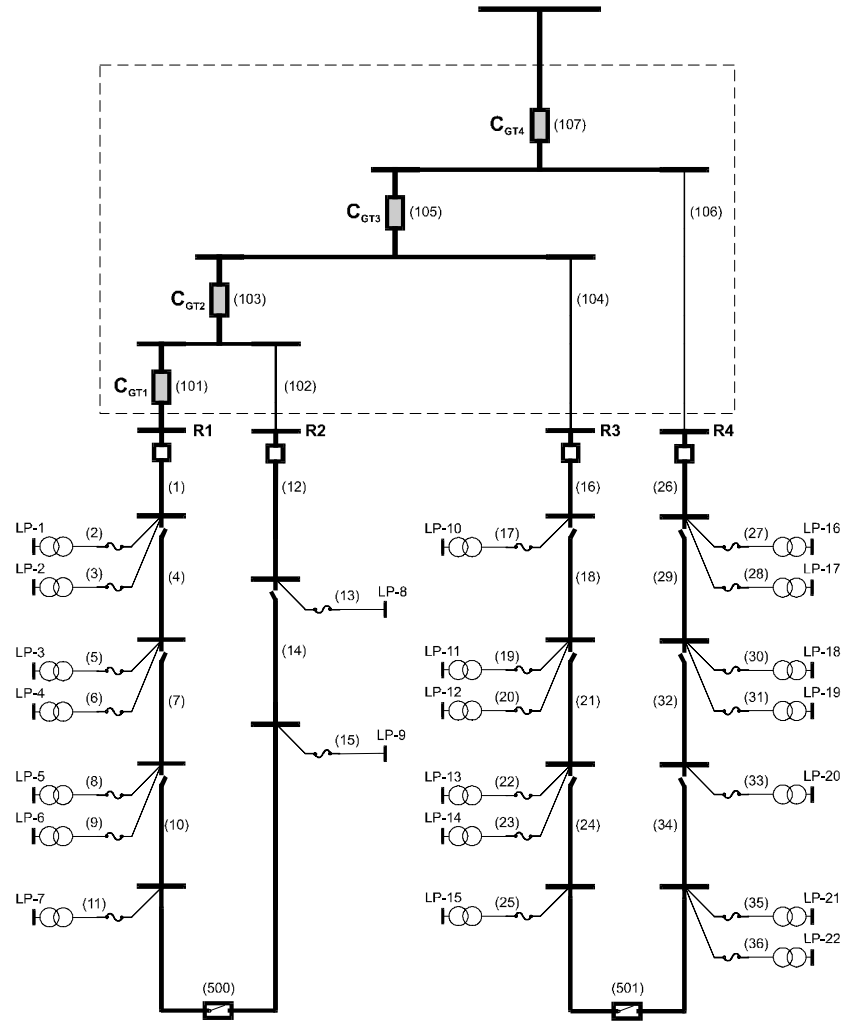

Figura 5: Sistema Elétrico de Potência Equivalente

RBTS-Barra2 e descritas em (Allan et alii, 1991). O sistema de potência completo e a rede equivalente para a avaliação NH3 estão representadas nas Figuras 4 e 5 .

\subsection{Análise NH3 para Carga Pico}

Esta análise considera apenas a situação de Carga Pico. Nesta condição, os equipamentos G\&T operam próximos à sua capa-cidade máxima, estando os consumidores mais sujeitos a interrupções.

Tabela 3: Parâmetros dos Componentes G\&T - Carga Pico

\begin{tabular}{|l|c|c|c|c|}
\hline \multirow{2}{*}{ Parâmetros } & \multicolumn{4}{|c|}{ Intervalo de Potência } \\
\cline { 2 - 5 } & $\mathbf{1}$ & $\mathbf{2}$ & $\mathbf{3}$ & $\mathbf{4}$ \\
\hline$\lambda[$ falhas/ano] & 9,609 & 2,939 & 3,159 & 4,763 \\
\hline $\mathbf{r}[$ horas $]$ & 35,646 & 39,366 & 46,256 & 28,360 \\
\hline $\mathbf{U}[$ horas/ano] & 342,562 & 115,724 & 146,153 & 135,08 \\
\hline
\end{tabular}

A Tabela 3 apresenta os parâmetros obtidos com a simulação Monte Carlo não-seqüencial para os componentes G\&T fictícios; os quais estão expressos em função de $\lambda$, $\mathrm{r}$ e $U$ associados com os intervalos de potência. Estes intervalos são definidos de acordo com a política de corte de carga e da capacidade de potência dos alimentadores princi-pais do sistema de distribuição, como mostrado 
Tabela 5: Índices para Pontos de Carga em Carga Pico

\begin{tabular}{|c|c|c|c|c|c|c|}
\hline & SISTEMA & FIC & DIC & r & EENS & LOLC \\
\hline \multirow{3}{*}{ LP-1 } & G\&T & 20,4730 & 739,6046 & 36,1259 & 641089,20 & 128217,80 \\
\cline { 2 - 7 } & Distrib. & 0,2442 & 3,5932 & 14,7114 & 3114,63 & 622,93 \\
\cline { 2 - 7 } & NH3 & 20,7172 & 743,1978 & 35,8734 & 644203,90 & 128840,80 \\
\hline \multirow{3}{*}{ LP-9 } & G\&T & 10,8630 & 397,0081 & 36,5468 & 743238,80 & 2378364,00 \\
\cline { 2 - 7 } & Distrib. & 0,1448 & 0,5098 & 3,5215 & 954,36 & 4636,49 \\
\cline { 2 - 7 } & NH3 & 11,0078 & 397,5178 & 36,1125 & 744193,10 & 2381418,00 \\
\hline \multirow{3}{*}{ LP-12 } & G\&T & 7,9230 & 281,2603 & 35,4992 & 205066,90 & 41013,37 \\
\cline { 2 - 7 } & Distrib. & 0,2605 & 3,6625 & 14,0588 & 2670,36 & 534,07 \\
\cline { 2 - 7 } & NH3 & 8,1835 & 284,9228 & 34,8167 & 207737,20 & 41547,45 \\
\hline \multirow{3}{*}{ LP-21 } & G\&T & 4,7630 & 135,0787 & 28,3600 & 123826,60 & 1077292,00 \\
\cline { 2 - 7 } & Distrib. & 0,2573 & 3,5943 & 13,9703 & 3294,93 & 28665,87 \\
\cline { 2 - 7 } & NH3 & 5,0203 & 138,6730 & 27,6225 & 127121,60 & 1105958,00 \\
\hline
\end{tabular}

na Tabela 4.

Tabela 4: Potência Pico dos ramais e Política de C.C.

\begin{tabular}{|c|c|c|c|}
\hline \multicolumn{2}{|c|}{$\begin{array}{c}\text { Potência do } \\
\text { Ramal }[\mathbf{M W}]\end{array}$} & $\begin{array}{c}\text { Corte de Carga } \\
\text { devido a G\&T }\end{array}$ & $\begin{array}{c}\text { Desconecta } \\
\text { o Ramal }\end{array}$ \\
\hline $\mathrm{R} 1$ & 5,93 & $0<C C_{G T} \leq 5,93$ & $\mathrm{R} 1$ \\
\hline $\mathrm{R} 2$ & 3,50 & $5,93<C C_{G T} \leq 9,43$ & $\mathrm{R} 1+\mathrm{R} 2$ \\
\hline $\mathrm{R} 3$ & 5,06 & $9,43<C C_{G T} \leq 14,5$ & $\mathrm{R} 1+\mathrm{R} 2+\mathrm{R} 3$ \\
\hline $\mathrm{R} 4$ & 5,51 & $14,5<C C_{G T} \leq 20,0$ & $\mathrm{R} 1+\mathrm{R} 2+\mathrm{R} 3+\mathrm{R} 4$ \\
\hline
\end{tabular}

Observa-se que o intervalo de potência 1 (de 0 a 5,934 MW), que corresponde ao Ramal 1, possui a maior taxa de falha $(\lambda)$. Este resultado era de se esperar, pois qualquer falha no sistema de alta tensão da Barra 6 do IEEERTS, retira de operação o Ramal 1. Nota-se também que a remoção de todos os 4 ramais (i.e. $\mathrm{R} 1+\mathrm{R} 2+\mathrm{R} 3+\mathrm{R} 4$, que corresponde ao intervalo de potência de 14,491 a 20,0 MW) possui a segunda maior taxa de falha, o que significa que a freqüência com que ocorrem estes cortes no sistema G\&T da Barra 6 é bastante significante.

A Tabela 5 exibe os índices de confiabilidade para os pontos de carga LP-1, LP-9, LP-12 e LP-21, que pertencem aos ramais $1,2,3$ e 4 , respectivamente. Os valores apresentados consideram os índices básicos FIC (falhas/ano), DIC (horas/ano), r (horas) mais os índices EENS (MWh/ano) e LOLC (US\$/ano). Todos os índices do NH3 são fornecidos na situação de carga pico e suas contribuições são desagregadas em G\&T e distribuição.

Ao analisar a Tabela 5, pode-se identificar claramente a política para cortes devido ao sistema G\&T. Observa-se que, a taxa de falha do Ramal 1 (igual a do LP-1) possui um valor maior (i.e. 20,473 falhas/ano) que a do Ramal 2 (i.e. 10,863 falhas/ ano) que, por sua vez, é maior que a do Ramal 3 e assim por diante, de acordo com a política de corte de carga adotada.
Tabela 6: Índices do Sistema para Carga Pico

\begin{tabular}{|c|c|c|c|c|}
\hline SISTEMA & FEC & DEC & EENS & LOLC \\
\hline G\&T & 11,1845 & 390,3521 & 7943191 & 36716580,00 \\
\hline Distrib. & 0,2532 & 3,6239 & 61607 & 304873,50 \\
\hline NH3 & 11,4377 & 393,9759 & 8004796 & 37018730,00 \\
\hline
\end{tabular}

Outro ponto que pode ser observado, é que a contribuição das falhas originadas do sistema G\&T é muito mais considerável que aquelas oriundas do sistema de distribuição. Deve-se salientar que, neste trabalho, o sistema G\&T é analisado por um algoritmo que utiliza fluxo de carga durante a simulação Monte Carlo, enquanto o desempenho do sistema de distribuição é avaliado pelo critério de continuidade.

A Tabela 6 apresenta os resultados obtidos para os índices do sistema, FEC (interrupções/consumidor ano), DEC (horas/ consumidor ano), EENS (MWh/ano) e LOLC (US\$/ano). Todos os índices NH3 obtidos na situação de carga pico também são desagregados nas contribuições devido aos sistemas G\&T e distribuição. Por exemplo, o índice FEC para todo o sistema (i.e. NH3) é igual a 11,4377 interrup-ções/consumidor, onde 11,1845 interrupções é procedente do sistema G\&T e somente 0,2532 interrupções origina-se do sistema de distribuição.

Analisando os resultados obtidos nas Tabelas 5 e 6 , pode-se concluir que o sistema de G\&T e o sistema de distribuição se comportam como dois componentes independentes conectados em série. A independência dos dois sistemas deve-se ao fato de se ter assumido que todos os equipamentos do sistema de distribuição (i.e. linhas, transformadores, etc.) são capazes de suportar a energia solicitada. Esta restrição, contudo, é devido ao critério de continuidade, que é amplamente utilizado na 
avaliação de sistemas de distribuição.

\subsection{Análise NH3 para Carga Média}

Esta análise considera a situação de carga média. Os equipamentos G\&T estão operando mais aliviados e, portanto, espera-se um desempenho muito melhor para o NH2. O procedimento descrito em 4.2 é repetido considerando a carga média, e os resultados para os índices do sistema são mostrados na Tabela 7 .

Tabela 7: Índices do Sistema para Carga Média

\begin{tabular}{|c|c|c|c|c|}
\hline SISTEMA & FEC & DEC & EENS & LOLC \\
\hline G\&T & 0,0200 & 15,3600 & 188789,8 & 876582,90 \\
\hline Distrib. & 0,2532 & 3,6239 & 37862,0 & 186782,00 \\
\hline NH3 & 0,2732 & 18,9839 & 226651,7 & 1061694,00 \\
\hline
\end{tabular}

Com o intuito de combinar os resultados obtidos com a carga Pico e Média, será suposto que a duração do pico diário de carga será de aproximadamente 1 hora. Portanto, se o conjunto dos índices anteriores forem ponderados nesta proporção, i.e. (1/24) para carga pico, e $(23 / 24)$ para carga média, que será denominado como carga fora de pico, será possível determinar um conjunto de índices equivalentes (EQ): e.g. o equivalente $\mathrm{FEC}_{G \& T}=0,4852$ e o equivalente $\mathrm{FEC}_{\text {Dist. }}=0,2532$ interrup-ções/consumidor ano. Assim, pode-se dizer que $65,71 \%$ das interrupções por consumidor são originadas no sistema G\&T, enquanto que $34,29 \%$ são oriundas do sistema de distribuição.

Podemos observar que neste exemplo, o impacto de falhas G\&T foi superior ao das falhas originadas no sistema de distribuição. Obviamente, este é um exemplo hipotético com a intenção de demonstrar o potencial da metodologia proposta, visto que em sistemas reais, como mencionado na Introdução, os sistemas de distribuição são responsáveis pela maior parte das contribuições individuais que acarretam indisponibilidade de fornecimento para os consumidores.

\subsection{Influência da Política de Corte de Carga}

Para avaliar a influência da política de corte de carga no nível de distribuição, a Tabela 8 apresenta uma nova política, onde o Ramal 3 é o primeiro a ser desconectado , seguido por R4, R1 e finalmente R2. Deve-se ressaltar que foi utilizada a mesma política para cortes no sistema G\&T. Portanto, não existe a necessidade de uma nova simulação no NH2. A única mudança é um remanejamento nos intervalos de potência no sentido de definir novos parâmetros fictícios para os componentes G\&T. A estrutura do sistema G\&T equivalente é a mesma mos- trada na Figura 5.

Tabela 8: Índices do Sistema para Carga Média

\begin{tabular}{|c|c|c|c|}
\hline \multicolumn{2}{|c|}{$\begin{array}{c}\text { Potência do } \\
\text { Ramal[MW] }\end{array}$} & $\begin{array}{c}\text { Corte de Carga } \\
\text { devido a G\&T }\end{array}$ & $\begin{array}{c}\text { Desconecta } \\
\text { o Ramal }\end{array}$ \\
\hline $\mathrm{R} 1$ & 5,93 & $0<C C_{G T} \leq 5,06$ & $\mathrm{R} 3$ \\
\hline $\mathrm{R} 2$ & 3,50 & $5,06<C C_{G T} \leq 10,6$ & $\mathrm{R} 3+\mathrm{R} 4$ \\
\hline $\mathrm{R} 3$ & 5,06 & $10,6<C C_{G T} \leq 16,5$ & $\mathrm{R} 3+\mathrm{R} 4+\mathrm{R} 1$ \\
\hline $\mathrm{R} 4$ & 5,51 & $16,5<C C_{G T} \leq 20,0$ & $\mathrm{R} 3+\mathrm{R} 4+\mathrm{R} 1+\mathrm{R} 2$ \\
\hline
\end{tabular}

A Tabela 9 apresenta os resultados para os índices do sistema obtidos com esta nova política de corte de carga. Comparando os resultados das Tabela 6 e 9, pode-se verificar que os índices relacionados com o sistema de distribuição não sofreram alterações. Isto ocorre porque a confiabilidade inerente ao sistema de distribuição depende somente das características das falhas de seus próprios componentes. Por outro lado, observa-se uma alteração nos índices referentes ao sistema G\&T e, consequentemente dos índices NH3. De fato, o sistema com esta nova política torna-se mais custoso do ponto de vista das interrupções.

Tabela 9: Índices do Sistema para Carga Pico

\begin{tabular}{|c|c|c|c|c|}
\hline SISTEMA & FEC & DEC & EENS & LOLC \\
\hline G\&T & 13,0544 & 463,3618 & 7828792 & 37539720,00 \\
\hline Distrib. & 0,2532 & 3,6239 & 61607 & 304873,50 \\
\hline NH3 & 13,3076 & 466,9857 & 7890399 & 37841880,00 \\
\hline
\end{tabular}

\section{CONCLUSÕES}

O sistema de distribuição realiza uma importante função dentro do fornecimento total de energia, pois providencia a conexão final entre as companhias de transmissão e seus consumidores. Em muitos países, o processo de privatização iniciou-se pelas companhias de distribuição. O novo modelo impõe uma mudança relevante em relação ao passado, onde a maioria das empresas de eletricidade era estatizada. A estrutura legal que se supõe ser a base para o ambiente competitivo idealizado para o setor elétrico, ainda não está totalmente implementada. Porém, as companhias de distribuição já estão sendo pressionadas pela opinião pública, e também pelas comissões e agências regula-doras, para melhorar a qualidade dos serviços contratados por seus consumidores. Com esta nova mentalidade, normas, incentivos, penalizações, responsabilidades etc. irão se tornar pontos fundamentais na discussão sobre o funcionamento dos sistemas elétricos de potência em todo o mundo.

A avaliação integrada da confiabilidade, incluindo geração, transmissão e distribuição, ou Nível Hierárquico 
3 (NH3), possui um importante papel no novo cenário competitivo, pois providencia uma visão mais abrangente do sistema em termos de desempenho passado ou futuro. O presente trabalho fornece uma contribuição na área da confiabilidade NH3, através do cálculo de índices, incluindo custos, que avaliam o desempe-nho total do sistema. A metodologia proposta é baseada na combinação da simulação de Monte Carlo com o conceito tradicional de minimum cut-set. Índices tradicionais, como FEC, DEC, etc. e também a LOLC, que representa o custo de interrupção, são desagregados considerando os níveis hierárquicos. Por fim, a metodologia proposta está sendo testada em sistemas de distribuição da CEMIG.

\section{REFERÊNCIAS}

Allan, R.N. and Da Silva, M.G. (1995). Evaluation of Reliability Indices and Outage Costs in Distribution Systems. IEEE Transactions on Power Systems, Vol. 10, No. 1, pp. 413-419.

Allan, R.N., Billinton, R., Sjarief, I., Goel, L., So, K.S. (1991). A Reliability Test System for Educational Purposes - Basic Distribution System Data and Results. IEEE Trans. on Power Systems, Vol. 6, No. 2, pp. 813-820.

Billinton, R. (1988). Distribution System Reliability Performance and Evaluation. Electrical Power and Energy Systems, Vol. 10, No. 3, pp. 190-200.

Billinton, R. and Alan, R.N. (1994). Reliability Evaluation of Power Systems. Plenum Press, NY, 2nd edition.

Billinton, R. and Allan, R.N. (1988). Reliability Assessment of Large Electric Power Systems. Kluwer Academic Pub., Boston.

Billinton, R. and Allan, R.N. (1992). Reliability Evaluation of Engineering Systems - Concepts and Techniques. Plenum Press, NY, 2nd edition.

Billinton, R. and Jonnavithula, S. (1996). A Test System for Teaching Overall Power System Reliability Assessment. IEEE Transactions on Power Systems, Vol. 11, No. 4, pp. 1670-1676.

Billinton, R. and Satish, J. (1996). Effect of Rotational Load Shedding on Overall Power System Adequacy Indices. IEE Proceedings, Part C, Vol. 143, No. 2 pp. 181-187.

Burns, S. and Gross, G. (1990). Value of Service Reliability. IEEE Transactions on Power Systems, Vol. 5, No.3, pp. 825-834.
Chowdhury, A.A. and Koval, D.O. (1998). Value-based Distribution System Reliability Planning. IEEE Transactions on Industry Applications, Vol. 34, No. 1, pp.23-29.

EPRI, Customer Demand for Service Reliability, Report RP-2801, 1989.

Goel, L. and Billinton, R. (1993). Utilization of Interrupted Energy Assessment Rates to Evaluate Reliability Worth in Electrical Power Systems. IEEE Transactions on Power Systems, Vol. 8, No. 3, pp. 929-936.

Leite da Silva, A.M., Cassula, A.M., Billinton, R., Manso, L.A.F. Integrated Reliability Evaluation of Generation, Transmission and Distribution Systems. IEE Proceed-ings, Part C, Vol. 149, No. 1, pp. 1-6.

Leite da Silva, A.M., Manso, L.A.F., Mello, J.C.O., Billinton, R. (2000). Pseudo-chronological Simulation for Composite Reliability Analysis with Time Varying Loads. IEEE Transactions on Power Systems, Vol. 15, No. 1, pp. 73-80.

Leite da Silva, A.M., Melo, A.C.G. and Cunha, S.H.F. (1991). Frequency and Duration Method for Reliability Evaluation of Large-scale Hydrothermal Generating Systems. IEE Proceedings, Part C, Vol. 138, No. 1, pp. 94-102.

Manso, L.A.F., Leite da Silva, A. M., Mello, J. C. O. (1999). Comparison of Alternative Methods for Evaluating Loss of Load Costs in Generation and Transmission System. Electric Power Systems Research, Vol. 50, Issue 2, pp. 107-114.

Mello, J. C. O., Leite da Silva, A. M., Pereira, M. V. F. (1997). Efficient Loss of Load Cost Evaluation by Combined Pseudo-sequential and State Transition Simulation. IEE Proceedings, Part C, Vol. 144, No. 2, pp. 147-154.

Mello, J.C.O., Pereira, M.V.F., Leite da Silva, A.M. (1994). Evaluation of Reliability Worth in Composite Systems Based on Pseudo-sequential Monte Carlo Simulation. IEEE Transaction on Power Systems, Vol. 9, No. 3, pp. 1318-1326.

Melo, A.C.G., Pereira, M.V. and Leite da Silva, A.M. (1993). A Conditional Probability Approach to the Calculation of Frequency and Duration Indices in Composite Reliability Evaluations. IEEE Transactions on Power Systems, Vol. 8, No. 3, pp. 11181125 
Task Force of the Application of Probability Methods Subcommittee. (1979). IEEE Reliability Test System. IEEE Transactions on Power Apparatus Systems., Vol. PAS-98, No. 6, pp. 2047-2054.

Wenyuan, Li and Billinton, R. (1993). A Minimum Cost Assessment Method for Composite Generation and Transmission System Expansion Planning. IEEE Trans. on Power Systems, Vol. 8, No. 2, pp. 628635. 